Full title: A Profile of Working Memory Ability in Poor Readers

Short title: Working Memory in Poor Readers

Journal name: Australian Psychologist

Authors: Dawes, E., Leitão, S., Claessen, M., \& Nayton, M.

Emily Dawes (PhD Candidate, Curtin University), Suze Leitão (Curtin University), Mary Claessen (Curtin University), Mandy Nayton (Dyslexia SPELD Foundation, Perth).

Correspondence: Emily Dawes

Email: emily.dawes@postgrad.curtin.edu.au

Research grants: Speech Pathology Australia (Undergraduate Research Grant, 2012) and Curtin University School of Psychology and Speech Pathology (ReCAP Grant, 2012).

\title{
Acknowledgements
}

Speech Pathology Australia and the Curtin University School of Psychology and Speech Pathology provided grants to fund this study. Thank you to the Dyslexia-SPELD Foundation and the schools, participants and families involved in the study. 


\begin{abstract}
Objective: This study aimed to provide a comprehensive working memory profile of a group of children with established poor reading ability.

Method: Participants included a group of established 'poor readers' and a group of ageand gender- matched controls with typically developing reading ability. The participants completed a comprehensive battery of assessments examining four components of working memory - the central executive, phonological loop, visuospatial sketchpad, and episodic buffer.
\end{abstract}

Results: As predicted, the poor reading group scored significantly lower than the typically developing reading group on measures of the phonological loop and central executive. There were no significant differences between the two groups on measures of the visuospatial sketchpad or episodic buffer. Contrary to predictions, a subgroup of poor readers with poor visuospatial working memory was not found, further highlighting the inconsistent findings in this area of working memory.

Conclusions: The results provide support for past research findings of deficits in the phonological loop and central executive of poor readers. The finding of typical episodic buffer functioning demonstrates the potential to draw on this relative strength in implementing interventions with poor readers. This implicates the importance of increasing awareness of specific working memory deficits in poor readers, and may guide future research into more effective teaching strategies and interventions for this population.

Keywords: Working memory, reading, poor readers, dyslexia, phonological loop, central executive. 
Reading is a complex cognitive process defined as the ability to use written text to extract and construct meaning (Vellutino, Fletcher, Snowling, \& Scanlon, 2004). As Vellutino et al., (2004, p. 5) state, “...one must be able to identify the words contained in running text with enough accuracy and fluency to allow computation of the meanings embodied in the text within the limits of working memory". Difficulties can arise during the process of learning to read, and many children continue to struggle with reading despite adequate instruction. Children who have a disability specific to reading may be diagnosed with dyslexia, characterised by specific difficulty in phonological processing and the decoding abilities necessary for efficient and accurate word recognition (Snowling, 2000). However, there is enormous discrepancy in diagnostic terminology, including dyslexia, reading disability and learning difficulty (Vellutino, et al., 2004). For the purpose of this research, the term 'poor readers' has been adopted.

Much research has focused on identifying the underlying deficit(s) in poor reading ability. It is thought that a core 'phonological' deficit is the basis of most poor readers' difficulties. This assumes weak phonological coding and, thus, difficulty in effectively mapping phonemes to graphemes during the process of learning to read and once reading is established (Vellutino, et al., 2004). A smaller group of poor readers who are thought to fit the 'surface' subtype of reading difficulty - display weaker skills in visual memory and/or processing speed, and present with poor sight word reading in the presence of intact grapheme-phoneme mapping (Snowling, 2000).

\section{Learning to Read}

During the process of learning to read, children are required to form connections between the visual symbols for letters and words, their phonemic and semantic representations, and their motor representation for articulation (Dehaene, 2009). Thus, 
reading is built on a foundation of accurate and stable phonological representations and an explicit knowledge of phonemes, and this phonemic awareness supports children to form crucial links between graphemes and phonemes in the early stages (Snowling, 2000). During the early development of decoding and word identification skills, children with poor phonological processing abilities, including phonemic awareness, rapid naming, and verbal working memory can be identified as at-risk (Snowling, 2000). Reading difficulties become increasingly pronounced after the age of 7 years, when an increased proportion of cognitive resources are allocated to encoding information linguistically, rather than visually (Gathercole \& Alloway, 2008). As such, many children are identified as poor readers in the middle years of primary schooling, as they transition from learning to read to reading to learn.

\section{Working Memory}

The process of learning to read is highly integrated with working memory, the capacity to temporarily retain and manipulate information (Gathercole \& Alloway, 2008). The multi-component subsystem model of working memory initially presented by Baddeley and Hitch (1974), and later revised, is well supported by research (Alloway, Gathercole, Willis, \& Adams, 2004). According to the revised model, working memory consists of four integral components (see Fig. 1). There are two 'slave' storage systems: the phonological loop temporarily stores phonological information and the visuospatial sketchpad temporarily maintains visual or spatial information (Baddeley \& Hitch, 1974). A third type of storage system, the episodic buffer, integrates or 'binds' and consequently stores information from the phonological loop and visuospatial sketchpad with long-term memory (Baddeley, 2000). These three storage systems work under the influence of the central executive, which is responsible 
for retrieving and manipulating information, and directing resources to the storage components (Baddeley \& Hitch, 1974). These four components function together to create 'working memory', allowing for the completion of many complex cognitive tasks such as reading.

"Insert Fig. 1 about here"

\section{Working Memory and Learning to Read}

The phonological loop is of particular interest in learning to read due to its role in learning new words (Baddeley, Gathercole, \& Papagno, 1998). Accurate and stable phonological representations of words must be maintained in order for the phonological loop to effectively hold and code novel phonological information, before assisting the conversion to long-term memory (Gathercole \& Baddeley, 1993). The phonological loop also supports the linking of phonological representations to matching graphemes, central in learning to read (Gathercole \& Baddeley, 1993). Thus, if the functioning or capacity of the phonological loop is inadequate, it is likely that the formation of accurate and stable phonological representations will be disrupted; adversely impacting a child's ability to establish the accurate grapheme-phoneme links necessary to develop fluent reading (Alloway et al., 2005).

The visuospatial sketchpad is also involved in the ability to read. The consistent visual information from graphemes must maintain a stable and accurate state in the visuospatial sketchpad while the appropriate phonological, semantic, and articulatory representations are accessed (Dehaene, 2009). A breakdown in the stability or accuracy of the graphemic material in the visuospatial sketchpad may lead to errors or dysfluency in reading. 
The central executive allows the manipulation of phonological information and, as such, a deficit may impact the appropriate manipulation and storage of phonological codes for the acquisition of language and literacy (Gathercole \& Baddeley, 1993). As a higher level component, difficulties in the central executive filter down and impact on processing in the phonological loop. Research has established a close link in the functioning of the phonological loop and central executive in typically developing children entering school (Alloway, et al., 2004) and in poor readers (Bayliss, Jarrold, Baddeley, \& Leigh, 2005).

Additionally, the central executive controls the episodic buffer, which is essential for reading development as it functions as a mediator between the visuospatial sketchpad, the phonological loop, and long-term memory (Baddeley, 2000). Access to information in long-term memory is necessary to support reading comprehension, when information from long-term memory is required to 'fill-in' missing visual, phonological or semantic information (Snowling, 2000). As the episodic buffer is a relatively new component of the working memory model, it has received limited research focus to date (Henry, 2010).

\section{Working Memory and Reading Difficulty}

Research has investigated the components of working memory in children with poor reading ability. Studies have found deficits in verbal span, a measure of the phonological loop, in children who are poor readers (Bayliss, et al., 2005; Roodenrys \& Stokes, 2001). In addition, 5 year old children with poor phonological loop functioning perform poorly compared to typically developing peers on literacy or language assessments at 8 years of age (Gathercole, Tiffany, Briscoe, \& Thorn, 2005). 
A meta-analysis examining the research into working memory, short-term memory, and reading disability identified 43 studies of children with reading disability, aged between 5 to 18 years, with significantly poor reading skills, but typical intelligence, as measured by standardised assessments (Swanson, Zheng, \& Jerman, 2009). The meta-analysis indicated persisting deficits in the phonological loop and central executive of children with reading disability (Swanson, et al., 2009). Evidence for a relationship between, and particular deficits in, the phonological loop and central executive of poor readers has become increasingly apparent (Gathercole, Alloway, Willis, \& Adams, 2006; Henry, 2012; Swanson, et al., 2009). While one study did not support a central executive deficit, it utilised a dual task (involving hand tapping) to assess this component, which may explain the discrepant findings (Kibby, Marks, Morgan, \& Long, 2004).

There has been some research into the visuospatial sketchpad skills of poor readers. Swanson et al. (2009) found that a portion of 'reading disabled' children exhibited visuospatial sketchpad deficits; however it was noted that the verbal component of the tasks selected could account for much of the variance. Deficits in visuospatial sketchpad and verbal span functioning have been reported in Italian children with dyslexia aged 8 to 13 years (Menghini, Finzi, Carlesimo, \& Vicari, 2011). In addition to poor phonological loop and central executive ability, Gathercole, et al., (2006) also found deficits in visuospatial sketchpad functioning, however participants in their study had poor maths ability as well as poor reading ability, and thus may have presented with a broader profile of learning difficulties. In contrast, other research has found typical visuospatial sketchpad functioning in children with dyslexia (Kibby, et al., 2004). 
As yet, there are no widely agreed upon measures used to assess functioning of the episodic buffer, and little evidence available regarding the functioning of the episodic buffer in children with dyslexia (Henry, 2012). One approach to measuring the episodic buffer is to use tasks that require binding of information in episodic memory, in other words requiring the integration, storage and retrieval of information (Henry, 2012). Binding tasks require the integration of verbal information, such as sentence repetition and story recall. Sentence repetition tasks are considered to draw on the integration of information from both the phonological loop (supporting recall of the words and their order in the sentences) and semantic and syntactic information retrieved from long-term memory, thus tapping into the episodic buffer (Alloway \& Gathercole, 2005). Sentence repetition tasks have been shown to be reliable markers of specific language impairment (Riches, 2012), and to be weaker in children with dyslexia (Plaza, Cohen \& Chevrie-Muller, 2002), although the findings have not generally been interpreted in relation to the episodic buffer. In an exploratory study which investigated episodic buffer functioning in children with intellectual disabilities (Henry, 2010), story recall was used as a measure to assess the binding of information from the phonological loop with representations from long term memory primed or activated by hearing the story. The children with intellectual disability did not differ from mental age-matched children in their recall, but performed significantly more poorly than children matched for age, supporting the notion that the episodic buffer functioning in this population was delayed rather than disordered (Henry, 2010). The results from a study by Roodenrys and Stokes (2001), which investigated verbal short-term memory, may also be interpreted in relation to the episodic buffer. In this study, poor readers exhibited similar performance to typically developing readers, who were reading age-matched, on 
memory span tasks for familiar words (Roodenrys \& Stokes, 2001). This suggested that there was no difference between the groups in the effects of material familiarity, and thus it could be interpreted that there was no difference between the groups in contributions from long-term memory via the episodic buffer. It is clear that tasks such as sentence repetition and story recall draw on and assess more than the episodic buffer. However, such tasks allow consideration of how information from long term memory is accessed and utilised during tasks requiring verbal binding.

Collectively, the research investigating the working memory of children who have poor reading ability has established that poor readers have deficits in the phonological loop, and there is increasing evidence for additional central executive deficits (Gathercole, et al., 2006; Swanson, et al., 2009). Varied findings exist regarding visuospatial sketchpad abilities in poor readers, with no specific body of evidence concerning the episodic buffer. There are equivocal findings regarding the specific nature of the deficit(s) and how these might change with age, in particular as children enter their teenage years.

Interpretation of findings is difficult, as no research has examined all four components of working memory in a single group of children. As such, the motivation of this study was to create a comprehensive profile of all four working memory components in one group of children with established poor reading ability, at the transition to secondary school.

\section{Hypotheses}

The research hypotheses were as follows:

1. The children identified as poor readers would have significantly lower performance on measures of the phonological loop and central executive. Past 
research has indicated a relationship between, and persisting deficits in, these areas of working memory in children with reading difficulty (Swanson, et al., 2009).

2. A subgroup of the children within the poor reading group would have significantly lower performance on visuospatial sketchpad tasks. Research findings are inconsistent in this area, however some research supports visuospatial sketchpad deficits in a subgroup of poor readers (Swanson, et al., 2009).

3. The children identified as poor readers would demonstrate similar performance to the group of typically developing readers on measures of the episodic buffer. The linguistic and semantic long-term knowledge of children who are established poor readers should compensate for weaker phonological loop skills (Henry, 2010).

\section{Method}

\section{Participants}

Ethical approval for the study was obtained from the Curtin University Human Research Ethics Committee and the Western Australian Department of Education. Poor readers. Potential participants for the poor reading group were identified and recruited with the assistance of the Dyslexia SPELD Foundation (DSF), Perth, Western Australia. The participants met the following criteria of 'poor reading':

- Typical or above average verbal comprehension ability as measured by the verbal comprehension index on the Wechsler Intelligence Scale for Children - Fourth Edition (WISC-IV) (Wechlser, 2003); 
- At least one composite score (i.e., phonological awareness, phonological memory, rapid naming) falling below the $25^{\text {th }}$ percentile on the Comprehensive Test of Phonological Processing (CTOPP) (Wagner, Torgesen \& Rashotte, 1999);

- A score below the $25^{\text {th }}$ percentile on the Reading component of the Wechsler Individual Achievement Test - Second Edition (WIAT-II) (i.e., word reading, nonsense word reading and reading comprehension) (Wechsler, 2007).

A total of 25 consent forms were received from the 76 parents who were approached, and 20 children completed the full assessment battery. The poor reading group $(n=20)$ consisted of 15 males and 5 females $\left(M_{\text {age }}=11.6\right.$ years, $S D=8.24$ months, age range: $10.4-13.1$ years).

Typically developing readers. A comparison group of 21 children identified as typically developing readers by their teachers were recruited from two primary schools in middle socio-economic areas of Perth, Western Australia.

Of the 95 parents who were approached, 43 consent forms were returned, and 21 children completed the full assessment battery. Potential participants who performed within one standard deviation of the mean or above on the Test of Word Reading Efficiency (TOWRE) (Torgesen, Wagner, \& Rashotte, 1999) were eligible for inclusion in the study.

The typically developing reading participants $(n=21)$ were age- and gendermatched to the poor reading group, consisting of 16 males and 5 females $\left(M_{\mathrm{age}}=11.9\right.$ years, $S D=10.75$ months, age range: $10.3-13.0$ years). An independent samples $t$ test indicated no significant difference in age between the groups, $t(39)=-1.32, p=.19$, two-tailed. There was no significant difference in gender between the groups using Fisher's Exact Test, $p=.60$, one-tailed. 


\section{Measures}

Test of Word Reading Efficiency (TOWRE) (Torgesen, et al., 1999). The TOWRE Form A is a timed reading assessment which measures the ability to efficiently and accurately read printed real words and non-words of increasing complexity (Torgesen, et al., 1999). Raw scores from sight word and phonemic decoding tasks are converted to standard scores $(M=100, S D=15)$.

Working Memory Test Battery for Children (WMTB-C) (Pickering \& Gathercole, 2001). The WMTB-C includes nine subtests that are designed to assess the functioning of the phonological loop, the visuospatial sketchpad, and the central executive, yielding component scores for these areas. Tasks used to assess the phonological loop included digit recall, word list recall, non-word list recall, and word list matching.

The visuospatial sketchpad tasks on the WMTB-C included block recall and memory for mazes. Central executive tasks included backwards digit recall, listening recall and counting recall.

Clinical Evaluation of Language Fundamentals - Fourth Australian Standardised Edition (CELF-4) (Semel, Wiig, \& Secord, 2003), and NEPSY Second Edition (NEPSY-II) (Korkman, et al., 2007). The recalling sentences subtest of the CELF-4 and the narrative memory subtest of the NEPSY Second Edition (NEPSY-II) (Korkman, Kirk, \& Kemp, 2007) were included as measures of the episodic buffer.

Non-word Repetition Task (Dollaghan \& Campbell, 1998). The non-word repetition task involves the participant hearing and verbally repeating non-words. The 16 non-words are presented in a progression of syllable lengths up to four syllables (Dollaghan \& Campbell, 1998). This task was included as an additional measure of the 
phonological loop as the WMTB-C non-word repetition subtest includes only single syllable non-words, and research indicates that non-word repetition tasks accurately differentiate children experiencing difficulty only at the 3 and 4 syllable level (Dollaghan \& Campbell, 1998). As per the task protocol, the non-words were audiorecorded by the primary researcher with a 2 to 3 second break between each non-word.

\section{Apparatus}

Portable audiometer. An Amplaid A137 audiometer with PELTOR air conduction headphones was used for hearing assessments. Participants were required to pass a hearing screen on the day of assessment, at a screening level of $25 \mathrm{~dB}$ between $500-4000 \mathrm{~Hz}$, to exclude the possibility of any hearing difficulties impacting on the assessment (Doyle, 1998).

Audio equipment. Three of the assessments (sentence recall, narrative recall and the non-word repetition task) were audio-recorded using a Memomic (XtremeMac) professional lapel microphone to allow for offline scoring. Praat (Boersma \& Weenink, 2010) acoustic analysis computer software was used with an Acer Aspire One Netbook to collect audio-recordings.

\section{Procedures}

As participants could not be randomly assigned to groups, a quasi-experimental equivalent control group research design was employed. Prior to completing the full assessment battery, the typically developing reading participants completed the TOWRE Form A. Data collection with the typically developing readers took place in a quiet room at their school. Data collection with the poor reading participants was conducted in a quiet room at their school or home. Informed written consent was 
obtained from all participants and their parents prior to assessment. The full assessment battery was completed in a single session of 60 to 90 minutes.

At the beginning of each assessment session, a hearing screen was completed. The WMTB-C was then administered, followed by the Dollaghan and Campbell (1998) non-word repetition task, recalling sentences subtest of the CELF-4, and the narrative memory subtest of the NEPSY-II. The latter three tasks were audio-recorded. Each assessment was administered following the guidelines in the respective article or manual (Dollaghan \& Campbell, 1998; Korkman, et al., 2007; Pickering \& Gathercole, 2001; Semel, et al., 2003). The administration of the tasks was counter-balanced in each group.

The WMTB-C subtests were scored on-line during assessments, and component scores were completed off-line by the primary researcher. Scoring of the non-word repetition task, sentence recall and narrative recall subtests were completed off-line by the primary researcher. Bivariate Pearson correlation coefficients were calculated to examine inter-rater reliability on $10 \%$ of the audio-recordings. Inter-rater reliability was acceptable for the aforementioned assessments, at $.95, .99$ and 1.00 , respectively. Scoring of the WMTB-C, CELF-4 sentence recall subtest, and NEPSY-II narrative recall subtest were completed according to test manual guidelines. Repetitions from the non-word repetition task were scored as correct or incorrect.

\section{Results}

The means and standard deviations of the working memory measures for the poor reading and typically developing reading groups are shown in Table 1. Means for both groups on the WMTB-C component scores $(M=100, S D=15)$ were within normal limits; however the group means of the poor readers were lower than the 
typically developing readers for all subtests. The means for the sentence recall and narrative recall subtests $(M=10, S D=3)$ were also within normal limits for both groups although the group mean for sentence recall for the poor readers was again lower than that of the typically developing readers.

"Insert Table 1 about here"

\section{Correlations between Measures of Working Memory}

The linear relationships between each of the working memory measures were evaluated across the whole sample using bivariate Pearson correlation coefficients. As expected, significant moderate to strong correlations were found between related working memory measures, displayed in Table 2 . Analyses were conducted in line with these correlations. Covariates were included in the episodic buffer analysis to reduce the influence of collinearity.

\section{Statistical Analyses}

In order to compare the performance of the two groups on measures of working memory, a series of independent measures multivariate analysis of variance (MANOVA) and univariate analysis of variance (ANOVA) tests were carried out. The results were analysed at an alpha level of .05 , unless otherwise reported. The effect size statistic reported is partial eta squared.

Prior to analysis, assumption testing was completed. Univariate normality was assumed for all measures in both groups. The homogeneity of variance assumption was violated for the phonological loop WMTB-C, visuospatial sketchpad WMTB-C, and episodic buffer sentence recall measures. Therefore results for these particular measures were analysed at a more conservative alpha level of .01. The multicollinearity and linearity assumptions were met for the two MANOVAs conducted, as correlations 
between the working memory measures were not excessive $(<.90)$ and the relationships between the measures were linear. The multivariate normality and homogeneity of variance-covariance assumptions were assumed for both MANOVAs.

\section{Phonological Loop and Central Executive}

As expected, there was a relationship between tasks measuring the phonological loop (the WMTB-C measure and the non-word repetition measure), and the central executive, demonstrating statistically significant, high correlation $(r(39)=.715, p<.01$ and $r(39)=.425, p<.01$, respectively). As such, a MANOVA was conducted with the two measures of the phonological loop and single measure of the central executive. A significant difference was found between the groups on a combination of these measures, $F(3,37)=7.880, p<.001, \mathrm{p} \eta^{2}=.390$, with the poor reading group means significantly lower than the typically developing reading group means. A statistically significant univariate ANOVA result was found for each individual measure. On the phonological loop WMTB-C measure, the poor reading group $(M=95.25)$ scored significantly lower than the typically developing reading group $(M=115.52), F(1,39)$ $=16.612, p<.001, \mathrm{p} \eta^{2}=.299$. The phonological loop non-word repetition task scores of the poor reading group $(M=9.35)$ also fell significantly below those of the typically developing reading group $(M=11.05), F(1,39)=6.140, p=.018, \mathrm{p} \eta^{2}=.136$. For the central executive WMTB-C measure, the poor reading group $(M=91.20)$ scored significantly lower than the typically developing reading group $(M=105.48), F(1,39)=$ 20.584, $p<.001, \mathrm{p} \eta^{2}=.345$. The reported $\mathrm{p} \eta^{2}$ values indicate large effects for the combination of measures and each individual measure.

\section{Visuospatial Sketchpad}


An ANOVA revealed no significant difference in visuospatial sketchpad scores between the poor reading group and the typically developing reading group with a small to medium effect, $F(1,39)=1.490, p=.23, \mathrm{p} \eta^{2}=.037$.

In order to explore whether a subgroup of extremely low visuospatial sketchpad scores appeared within the poor reading group, a statistical method developed by Crawford and Howell (1998) was used. This created individual point estimates, or effect sizes, for each participant within the group. The approach treats the normative sample, the visuospatial sketchpad scores of the poor readers, as statistics rather than population parameters, to obtain effect sizes for the abnormality of the difference of each individual score, as compared to the group mean (Crawford \& Howell, 1998). The effect sizes and confidence limits for each visuospatial sketchpad score in the poor reading group are shown in Table 3. All effect sizes were within the typical range (within +/- 3).

\section{"Insert Table 3 about here"}

\section{Episodic Buffer}

A MANOVA was used to compare the two groups on the episodic buffer measures of sentence recall and narrative recall. Due to collinearity between the phonological loop and central executive measures of the WMTB-C and the sentence recall task $(r(39)=$ $.530, p<.01$ and $r(39)=.565, p<.01$, respectively), the two former measures were included as covariates in the analysis. The MANOVA was not statistically significant, $F$ $(2,36)=0.138, p=.87, \mathrm{p} \eta^{2}=.008$, indicating no significant difference between the groups on episodic buffer measures. The reported $\mathrm{p} \eta^{2}$ value indicates a small effect. Although nonsignificant, consideration of the individual tasks demonstrates a greater difference between the sentence recall task for the poor reading group $(M=8.5)$ and the 
typically developing reading group $(M=10.00)$, than for the narrative recall task $(M=$ 9.80 and 10.05 respectively).

\section{Discussion}

The present study aimed to provide a comprehensive working memory profile of a group of established 'poor readers' compared to a group of typically developing readers, as they approached secondary school age, a significant stage in their educational pathway. The first hypothesis of the study was confirmed, as the poor readers performed significantly more poorly on measures of the phonological loop and the central executive. The second hypothesis was not confirmed, as a subgroup of poor readers with significantly lower visuospatial sketchpad functioning was not found. The finding of generally typical episodic buffer functioning in poor readers supported the third hypothesis of the study.

\section{Phonological Loop and Central Executive}

As hypothesised, the poor readers scored significantly lower than the typically developing readers on measures of the phonological loop and the central executive. The large effect sizes for each measure reflect the high amount of variance between the groups attributable to the effect of the phonological loop (WMTB-C $\mathrm{p} \eta^{2}=.299$, nonword repetition $\left.\mathrm{p} \eta^{2}=.136\right)$ and the central executive $\left(\mathrm{WMTB}-\mathrm{C} \mathrm{p} \eta^{2}=.345\right)$. As described in the literature, there was a high correlation between the phonological loop and central executive tasks, indicating a strong interrelation between these two components of working memory. All tasks used to assess the central executive in this study were verbal. It is therefore questionable as to whether the tasks used to assess the phonological loop, and those used to assess the central executive, accurately differentiated enough between the two components of working memory. However, this 
correlation was expected, and the results of tasks assessing these two components of working memory were analysed and considered together.

Interestingly, the mean WMTB-C component scores of the poor readers on the phonological loop $(M=95.25)$ and central executive $(M=91.20)$ fell within normal limits. As such, their scores would generally be considered 'typical'. However, the poor reading group mean scores for both measures were significantly lower than those of the typically developing readers, indicating that the poor readers in this study have less robust phonological loop and central executive skills to support their reading development than their typically developing reading peers. Critically, the phonological loop is imperative for language learning (Baddeley, et al., 1998), and poor vocabulary development has been identified in infants at high-risk of dyslexia (Koster et al., 2005). Previous literature has found that poor phonological loop functioning at a young age persists, and is linked to later literacy and language difficulties (Gathercole, et al., 2005). As working memory capacity does not develop significantly after the age of 15 years, a deficit present in upper primary school will have a direct impact on the efficient use of phonological codes in reading, and hence, on language learning through reading (Gathercole \& Alloway, 2008). Likewise, the central executive interacts with the phonological loop and allows for the online manipulation of phonological information. Thus, difficulties here will have a direct adverse impact upon reading, particularly when the manipulation of phonological units is required to decode, hold in memory, and learn, a novel word. The current findings support previous research, indicating that working memory difficulties in these components are inherent in poor readers.

Such underlying difficulties would indicate that the transition from relying primarily on the visuospatial sketchpad to the phonological loop at around 7 years of 
age, characteristic of typically developing readers, is an ineffective way for poor readers to maximise the efficiency of their working memory in reading (Gathercole \& Alloway, 2008). This is reflected in the fact that reading difficulty is usually recognised, and often becomes more pronounced, after this age (Gathercole \& Alloway, 2008).

In addition, poor phonological loop and central executive functioning may be an underlying factor in the core difficulty for many poor readers: weak phonological coding (Vellutino, et al., 2004). Specifically, this relates to accurately mapping alphabetic symbols to sounds so that they can be retrieved and manipulated efficiently within the constraints of the phonological loop and central executive. As such, weaknesses may have a direct and significant impact on this mapping and retrieval process, causing difficulty in the process of learning to read and reading.

Poor phonological loop functioning does not negate the use of auditory support in teaching poor readers, given the average or above average verbal comprehension of much of this population. Reducing the overall length and complexity of written material would lessen demands on the phonological loop and the central executive of poor readers (Gathercole \& Alloway, 2008). Providing auditory reinforcement of written material, in addition to the written material itself, may facilitate reading and, by utilising the auditory pathway, improve reading comprehension. Improved reading comprehension has been demonstrated in children with dyslexia using this approach (Elkind, Cohen, \& Murray, 1993).

\section{Visuospatial Sketchpad}

This study found no significant difference between the visuospatial sketchpad scores of the poor reading and the typically developing reading groups. This was supported by the small effect size $\left(\mathrm{p} \eta^{2}=.037\right)$, indicating that group accounted for a 
relatively small amount of variance in visuospatial sketchpad ability. This finding supports the body of research negating the theory of visuospatial sketchpad deficits in poor readers (Kibby, et al., 2004).

In contrast to the hypothesised predictions, a subgroup of poor readers with significantly low visuospatial sketchpad scores did not emerge in the data. Only one of the poor reading participants' visuospatial sketchpad scores was close to extreme (Participant 3, Zcc $=-2.53$ ), indicating that the poor readers in this study generally possessed typical visuospatial sketchpad ability. It is possible that this reflects the nature of reading deficits in the poor reading participants as falling into the 'phonological' rather than 'surface' form of difficulty (Snowling, 2000). The 'surface' form, involving visual memory and/or processing speed, is reported in a minority of poor readers, and therefore it would be pertinent to replicate these results with a larger sample (Snowling, 2000).

Visuospatial sketchpad deficits have been reported in Italian children with dyslexia (Menghini, et al., 2011). The transparent mapping of phonemes to graphemes in Italian orthography dictates that learning to read in Italian is generally a rapid and undemanding process, comparative to English (Cossu, 1999). Hence, it is possible that children learning to read in Italian only present with reading difficulty if they have the 'surface' form of reading difficulty; or alternatively, if deficits in both the phonological loop and visuospatial sketchpad are present (Menghini, et al., 2011). This would imply that, due to the transparency of the orthography, Italian children with poor phonological loop and central executive ability are able to use typical visuospatial sketchpad ability to overcome poor reading in contrast to children learning to read complex English orthography. 
Past research has found visuospatial sketchpad deficits in children with poor reading and mathematical abilities (Alloway, et al., 2009; Gathercole, et al., 2006). It is likely that the numbers and figures involved in learning and performing maths utilise visuospatial sketchpad resources, and draw on central executive function to an extent, but less on the phonological loop (Alloway \& Passolunghi, 2011). Hence, maths ability may have been a confounding factor in these studies, and consequently only children with very poor skills in maths, in addition to reading, presented with deficient visuospatial sketchpad functioning (Alloway \& Passolunghi, 2011).

Swanson et al. (2009) queried whether visuospatial sketchpad functioning in poor readers declines with increased processing demands. However, increased information processing demands would implicate the central executive, and thus a decline in visuospatial sketchpad function is more likely to pertain to deficient central executive functioning than poor visuospatial sketchpad ability alone. This viewpoint is supported by Smith-Spark, Fisk, Fawcett and Nicolson (2003), who found that adults with dyslexia demonstrated poorer performance than controls on visuospatial sketchpad tasks when the central executive was implicated, that is, when the processing demands of the task became very high.

The finding of similar performance across groups in this study provides support for reading as a primarily linguistic, rather than visual, process. This implies that, although visuospatial sketchpad abilities are used in reading, it is not poor visuospatial sketchpad ability which underlies or maintains poor reading for most poor readers. This result has significant implications for interventions used to assist poor readers, in that interventions must focus on the phonological components of reading. In addition, typical visuospatial functioning may be utilised as a strength by employing strategies to 
support poor readers (e.g. the use of visual supports may improve reading comprehension). However, it is important to replicate these results with a larger group of poor readers to provide further support for this finding.

\section{Episodic Buffer}

As predicted, there was no significant difference between the poor reading and typically developing reading groups on measures of the episodic buffer, with a small effect size $\left(\mathrm{p} \eta^{2}=.008\right)$. This result confirms the hypothesis that poor readers have typical linguistic and semantic knowledge in long-term memory, accessed via the episodic buffer (Henry, 2010).

However, it is pertinent to discuss the collinearity between the episodic buffer sentence recall task, and the phonological loop and central executive tasks of the WMTB-C. It is evident from the reported correlations that the demands of the sentence recall task were closely related to the demands of the phonological loop and central executive WMTB-C tasks, whereas those of the narrative recall task were not. This highlights the issue that tasks which require an individual to establish connections between items, store and retrieve verbal information will by necessity draw on several areas of knowledge (such as vocabulary and grammar) as well as draw on phonological loop resources. It is thus difficult to establish a 'pure' measure of the episodic buffer. This is also reflected in the consideration of the individual task means for the two episodic buffer measures, which demonstrated a greater difference for the sentence recall task. Potentially, the greater influence of the phonological loop and/or central executive on the sentence recall task, as reflected in the task correlations, may explain this difference in group means between the two episodic buffer tasks. 
Theoretically, narrative recall may be a purer measure of episodic buffer ability than sentence recall. Rather than pooling a portion of resources through the phonological loop, narrative recall relies heavily on using long-term linguistic and semantic knowledge to maintain a schema for recall and fill-in missing information (Henry, 2010). The scoring guidelines for the two episodic buffer measures reflected this difference, as sentence recall required exact repetition, whereas narrative recall required key terms and a summary of information. The sentence recall task utilises the episodic buffer for long-term linguistic knowledge of sentence structure, however also accesses the phonological loop, under the control of the central executive, in order to integrate temporary phonological representations into sentence structure for accurate repetition (Riches, 2012; Willis \& Gathercole, 2001). These findings support research that has found an association between the episodic buffer, and the phonological loop and central executive (Alloway, et al., 2004). In addition, the results highlight the need for further research into the validity of tasks used to assess and explore the episodic buffer. Future research should extend the range of tasks and include measures such as those that assess cross-modal binding, given the recent findings of difficulties allocating attentional resources in such tasks by dyslexic readers (Jones, Branigan, Parra \& Logie, 2013).

As the aforementioned correlations were controlled for using covariates in the analysis, the findings of this study provide direct evidence of typical episodic buffer functioning in a group of poor readers. Semantic, rather than phonological, skills have a greater influence on readers during high school, due to the need to comprehend written information and critically, in order to 'read to learn' (Vellutino, et al., 2004). Providing support to processing in the episodic buffer may decrease pressure on the poor 
functioning of the phonological loop and central executive, and maximise learning opportunity and retention of information for poor readers.

The episodic buffer, in addition to accessing long-term memory, binds information together in 'chunks' (Baddeley, 2000). Presenting written information in small amounts and enabling children to verbally rephrase information would allow for 'chunking' in the episodic buffer, and potentially improve reading comprehension (Gathercole \& Alloway, 2008). In this way, the processing load on both the phonological loop and central executive would be lessened, as poor readers would focus processing on the episodic buffer to integrate and understand written material (Baddeley, Hitch, \& Allen, 2009).

\section{Limitations}

The poor reading participants were identified as being eligible for this study on the basis of cut-off scores from a number of assessments completed at the Dyslexia SPELD Foundation, used to diagnose eligibility for their services. As such, the current reading ability of the poor readers was not assessed for this study. Individual reading ability scores of the poor readers would have enabled further analysis of the findings, such as investigating whether certain components of working memory may predict reading ability in poor and / or typically developing readers. Future research would benefit from such data collection and analysis.

Additionally, information about reading intervention/s received by the participants was not collected. This would have provided valuable information, enabling analysis to identify potential differences related to level of intervention received. However, this 'blindness' also allowed the study to provide strong evidence for an 
implicit pattern of working memory deficits and strengths across the poor reading group.

\section{Conclusion}

This study has provided a comprehensive profile of working memory in a group of children with established poor reading ability, at the point of transition to secondary school. It has both confirmed and advanced previous research, providing evidence for specific difficulties in the phonological loop and central executive of poor readers. This study also presented evidence for typical visuospatial sketchpad functioning in this group of poor readers, supporting the notion of reading difficulty as predominantly a phonologically based problem. Furthermore, the finding of generally typical episodic buffer functioning in poor readers is of significance. Future research could investigate how to effectively incorporate compensatory strategies and modify teaching and intervention to support processing in identified areas of relative working memory strength for poor readers, particularly to support reading comprehension at the transition to highschool. The findings will assist psychologists, teachers and speech pathologists to support the learning needs of poor readers as they enter secondary school by decreasing demand on weak working memory components, and supporting processing in areas of relative working memory strength. Finally, this study provides justification to increase awareness of working memory deficits in poor readers and direct future research towards developing effective, evidence-based teaching strategies and interventions for poor readers. 


\section{Key Points}

\section{What is already known about this topic:}

- Working memory is highly involved in learning to read and the process of reading.

- Many children who are poor readers demonstrate poor phonological processing.

- Poor readers generally demonstrate deficits in the working memory areas involved in processing linguistic information (the phonological loop) and manipulating information (the central executive), but findings regarding other areas of working memory are inconsistent.

\section{What this paper adds:}

- This study provides the first complete profile of working memory in one group of children with established poor reading ability. The study confirms previous research findings of significantly poor performance in the phonological loop and central executive areas of working memory in poor readers.

- The poor reading group demonstrated typical visuospatial sketchpad functioning, and therefore their reading difficulty could not be attributed to poor visuospatial working memory skills.

- The poor reading group demonstrated typical episodic buffer functioning, indicating that access to long-term semantic representations was an area of relative working memory strength. This result has implications for strategies to support reading and reading comprehension in poor readers. 


\section{References}

Alloway, T. P., Gathercole, S. E., Adams. (2005). The role of sentence recall in reading and language skills of children with learning difficulties. Learning \& Individual Differences, 15, 271-282. doi: 10.1016/j.lindif.2005.05.001

Alloway, T. P., Gathercole, S. E., Adams, A.-M., Willis, C., Eaglen, R., \& Lamont, E. (2005). Working memory and phonological awareness as predictors of progress towards early learning goals at school entry. British Journal of Developmental Psychology, 23(3), 417-426. doi: 10.1348/026151005X26804

Alloway, T. P., Gathercole, S. E., Kirkwood, H., \& Elliott, J. (2009). The Cognitive and Behavioral Characteristics of Children With Low Working Memory. Child Development, 80(2), 606-621. doi: 10.1111/j.1467-8624.2009.01282.x

Alloway, T. P., Gathercole, S. E., Willis, C., \& Adams, A.-M. (2004). A structural analysis of working memory and related cognitive skills in young children. Journal of Experimental Child Psychology, 87(2), 85-106. doi: 10.1016/j.jecp.2003.10.002

Alloway, T. P., \& Passolunghi, M. C. (2011). The relationship between working memory, IQ, and mathematical skills in children. Learning and Individual Differences, 21(1), 133-137. doi: 10.1016/j.lindif.2010.09.013

Baddeley, A. (2000). The episodic buffer: a new component of working memory? Trends in Cognitive Sciences, 4(11), 417-423. doi: 10.1016/S13646613(00)01538-2

Baddeley, A., Gathercole, S., \& Papagno, C. (1998). The phonological loop as a language learning device. Psychological Review, 105(1), 158-173. doi: 10.1037/0033-295X.105.1.158 
Baddeley, A., \& Hitch, G. (1974). Working memory. In G. H. Bower (Ed.), The Psychology of Learning and Motivation (Vol. 8, pp. 48-79). New York: Academic Press.

Baddeley, A., Hitch, G., \& Allen, R. (2009). Working memory and binding in sentence recall. Journal of Memory and Language, 61(3), 438-456. doi: 10.1016/j.jml.2009.05.004

Bayliss, D. M., Jarrold, C., Baddeley, A., \& Leigh, E. (2005). Differential constraints on the working memory and reading abilities of individuals with learning difficulties and typically developing children. Journal of Experimental Child Psychology, 92(1), 76-99. doi: 10.1016/j.jecp.2005.04.002

Bialystok, E. (2007). Cognitive Effects of Bilingualism: How linguistic experience leads to cognitive change. The International Journal of Bilingual Education and Bilingualism, 10(3), 210-223. doi: 10.2167/beb441.0

Boersma, P., \& Weenink, D. (2010). Praat: doing phonetics by computer [Computer program] (Version 5.1.44). Retrieved from http://www.praat.org/

Cohen, J. (1992). A Power Primer. Psychological Bulletin, 112(1), 155-159. doi: $10.1037 / 0033-2909.112 .1 .155$

Cossu, G. (1999). The acquisition of Italian orthography. In M. Harris \& G. Hatano (Eds.), Learning to Read and Write: A cross-linguistic perspective (pp. 10-33). Cambridge: Cambridge University Press.

Cowan, N. (1988). Evolving Conceptions of Memory Storage, Selective Attention, and their Mutual Constraints within the Human Information-Processing System. Psychological Bulletin, 104(2), 163-191. doi: 10.1037/0033-2909.104.2.163 
Crawford, J. R., \& Howell, D. C. (1998). Comparing an Individual's Test Score Against Norms Derived from Small Samples. The Clinical Neuropsychologist, 12(4), 482-486. doi: 10.1076/clin.12.4.482.7241

Dehaene, S. (2009). Reading in the Brain. New York: Penguin Group.

Dollaghan, C., \& Campbell, T. F. (1998). Nonword Repetition and Child Language Impairment. Journal of Speech, Language \& Hearing Research, 41, 1136-1146. doi: 10.1044 jslhr.4105.1136

Doyle, J. (1998). Practical Audiology for Speech-Language Therapists. London: Whurr Publishers.

Elkind, J., Cohen, K., \& Murray, C. (1993). Using computer-based readers to improve reading comprehension of students with dyslexia. Annals of Dyslexia, 43(1), 238-259. doi: 10.1007/BF02928184

Gathercole, S. E., \& Alloway, T. P. (2008). Working Memory \& Learning. London: SAGE Publications.

Gathercole, S. E., Alloway, T. P., Willis, C., \& Adams, A. M. (2006). Working memory in children with reading disabilities. Journal of Experimental Child Psychology, 93(3), 265-281. doi: 10.1016/j.jecp.2005.08.003

Gathercole, S. E., \& Baddeley, A. D. (1993). Working Memory and Language. Hove: Lawrence Erlbaum Associates.

Gathercole, S. E., Tiffany, C., Briscoe, J., \& Thorn, A. (2005). Developmental consequences of poor phonological short-term memory function in childhood: a longitudinal study. Journal of Child Psychology and Psychiatry, 46(6), 598-611. doi: 10.1111/j.1469-7610.2004.00379.x 
Glazzard, J. (2010). The impact of dyslexia on pupils' self-esteem. Support for Learning, 25(2), 63-69. doi: 10.1111/j.1467-9604.2010.01442.x

Henry, L. (2010). The episodic buffer in children with intellectual disabilities: An exploratory study. Research in Developmental Disabilities, $31(6$ - 3), 16091614. doi: 10.1016/j.ridd.2010.04.025

Henry, L. (2012). The Development of Working Memory in Children. London: SAGE Publications.

Jean, M., \& Geva, E. (2009). The development of vocabulary in English as a second language children and its role in predicting word recognition ability. Applied Psycholinguistics, 30(1), 153 - 186. doi: 10.1017/S0142716408090073

Jones, M. W., Branigan, H. P., Parra M. A., \& Logie, R. H. (2013). Cross-modal binding in developmental dyslexia. Journal of Experimental Psychology: Learning, Memory and Cognition. Advance online publication. doi: $10.1037 / \mathrm{a} 0033334$

Just, M. A., \& Carpenter, P. A. (1992). A Capacity Theory of Comprehension: Individual differences in working memory. Psychological Review, 99(1), 122149. doi: $10.1037 / 0033-295 X .99 .1 .122$

Kibby, M. Y., Marks, W., Morgan, S., \& Long, C. J. (2004). Specific Impairment in Developmental Reading Disabilities: A working memory approach. Journal of Learning Disabilities, 37(4), 349 - 363. doi: 10.1177/00222194040370040601

Korkman, M., Kirk, U., \& Kemp, S. (2007). NEPSY - Second Edition (NEPSY- II). San Antonio: TX: Pearson.

Koster, C., Been, P. H., Krikhaar, E. M., Zwarts, F., Diepstra, H. D., \& van Leeuwen, T. H. (2005). Differences at 17 Months: Productive language patterns in infants at 
familial risk for dyslexia and typically developing infants. Journal of Speech, Language, and Hearing Research, 48(2), 426-438. doi: 10.1044/1092$4388(2005 / 029)$

Menghini, D., Finzi, A., Carlesimo, G. A., \& Vicari, S. (2011). Working Memory Impairment in Children With Developmental Dyslexia: Is it Just a Phonological Deficity? Developmental Neuropsychology, 36(2), 199-213. doi: $10.1080 / 87565641.2010 .549868$

Pickering, S., \& Gathercole, S. (2001). The Working Memory Test Battery for Children (WMTB-C). London: Pearson Assessment.

Plaza, M., Cohen, H., \& Chevrie-Muller, C. (2002). Oral language deficits in dyslexic children: Weaknesses in working memory and verbal planning. Brain and Cognition, 48, 505-512. doi: 10.1006/brcg.2001.1407

Riches, N. G. (2012). Sentence Repetition in Children with Specific Language Impairment: An investigation of underlying mechanisms. International Journal of Language \& Communication Disorders, 47(5), 499-510. doi: 10.1111/j.14606984.2012.00158.x

Roodenrys, S., \& Stokes, J. (2001). Serial recall and nonword repetition in reading disabled children. Reading and Writing: An Interdisciplinary Journal, 14, 379394. doi: 10.1023/A:1011123406884

Semel, E., Wiig, E., \& Secord, W. (2003). Clinical Evaluation of Language Fundamentals - Australian Standardised (Fourth ed.). Marrickville: NSW: Harcourt Assessment.

Smith-Spark, J., Fisk, J., Fawcett, A. \& Nicolson, R. (2003). Investigating the central executive in adult dyslexics: Evidence from phonological and visuospatial 
working memory performance. European Journal of Cognitive Psychology, 15(4), 567-587. doi: 10.1080/09541440340000024

Snowling, M. J. (2000). Dyslexia (2nd ed.). Oxford: Blackwell Publishers.

Swanson, H. L., Zheng, X., \& Jerman, O. (2009). Working Memory, Short-Term Memory, and Reading Disabilities: A selective meta-analysis of the literature. Journal of Learning Disabilities, 42(3), 260-287. doi: $10.1177 / 0022219409331958$

Torgesen, J., Wagner, R., \& Rashotte, C. (1999). Test of Word Reading Efficiency (TOWRE): Pearson Assessment.

Vellutino, F. R., Fletcher, J. M., Snowling, M. J., \& Scanlon, D. M. (2004). Specific reading disability (dyslexia): what have we learned in the past four decades? Journal of Child Psychology and Psychiatry, 45(1), 2-40. doi: 10.1046/j.00219630.2003.00305.x

Wagner, R., Torgesen, J., \& Rashotte, C. (1999). Comprehensive Test of Phonological Processing (CTOPP) Austin, TX: PRO-ED.

Wechsler, D. (2007). Wechsler Individual Achievement Test - Second Edition, Australian Standardised Edition (WIAT-II Australian). London: The Psychological Corporation.

Wechsler, D. (2003). Wechsler Intelligence Scale for Children - Fourth Edition (WISCIV). London: Pearson Assessment.

Willis, C. S., \& Gathercole, S. E. (2001). Phonological short-term memory contributions to sentence processing in young children. Memory, 9, 349-363. doi: $10.1080 / 09658210143000155$ 


\section{Tables}

Table 1

Means and Standard Deviations of Working Memory Measures

\begin{tabular}{|c|c|c|c|c|}
\hline & \multicolumn{2}{|c|}{ Poor readers $(n=20)$} & \multicolumn{2}{|c|}{$\begin{array}{l}\text { Typically Developing } \\
\text { readers }(n=21)\end{array}$} \\
\hline & Mean & $\begin{array}{l}\text { Standard } \\
\text { Deviation }\end{array}$ & Mean & $\begin{array}{l}\text { Standard } \\
\text { Deviation }\end{array}$ \\
\hline Central Executive - WMTB-C & 91.20 & 14.19 & 105.48 & 19.51 \\
\hline Phonological Loop - WMTB-C & 95.25 & 12.06 & 115.52 & 18.87 \\
\hline Visuo-spatial Sketchpad - WMTB-C & 91.10 & 11.89 & 97.14 & 18.85 \\
\hline Phonological Loop - Non-word & 9.35 & 1.93 & 11.05 & 2.42 \\
\hline repetition & & & & \\
\hline Episodic Buffer - Sentence recall & 8.40 & 1.54 & 10.00 & 3.09 \\
\hline Episodic Buffer - Narrative recall & 9.80 & 3.23 & 10.05 & 2.69 \\
\hline
\end{tabular}


Table 2

Correlations (Pearson's r) between Measures of Working Memory

\begin{tabular}{|c|c|c|c|c|}
\hline \multirow[t]{3}{*}{ Measure } & Phonological & Phonological & Central & Episodic \\
\hline & Loop - & Loop - Non-word & Executive - & Buffer - \\
\hline & WMTB-C & repetition & WMTB-C & Sentence recall \\
\hline \multicolumn{5}{|l|}{ Phonological Loop - } \\
\hline \multicolumn{5}{|l|}{ WMTB-C } \\
\hline Phonological Loop - & $.387 *$ & & & \\
\hline \multicolumn{5}{|l|}{ Non-word repetition } \\
\hline Central Executive - & $.715^{* *}$ & $.425 * *$ & & \\
\hline \multicolumn{5}{|l|}{ WMTB-C } \\
\hline Episodic Buffer - & $.530 * *$ & .243 & $.565 * *$ & \\
\hline \multicolumn{5}{|l|}{ Sentence recall } \\
\hline Episodic Buffer - & .032 & .010 & .067 & $.341^{*}$ \\
\hline Narrative recall & & & & \\
\hline
\end{tabular}

Note: * Correlation is significant at the .05 level; ** Correlation is significant at the .01 level. 
Table 3

Effect Sizes (Zcc) and Confidence Limits of the Poor Reading Participants Visuo-spatial Sketchpad Scores

\begin{tabular}{|c|c|c|}
\hline Participant & $\mathrm{Z} c c$ & 95\% Confidence Limits \\
\hline 1 & 1.001 & 0.452 to 1.533 \\
\hline 2 & 1.422 & 0.785 to 2.040 \\
\hline 3 & -2.533 & -3.436 to -1.613 \\
\hline 4 & -0.345 & -0.792 to 0.111 \\
\hline 5 & -0.766 & -1.258 to -0.257 \\
\hline 6 & -0.345 & -0.792 to 0.111 \\
\hline 7 & 0.749 & 0.243 to 1.239 \\
\hline 8 & -0.093 & -0.531 to 0.348 \\
\hline 9 & -0.682 & -1.163 to -0.186 \\
\hline 10 & 1.590 & 0.915 to 2.247 \\
\hline 11 & 1.170 & 0.587 to 1.734 \\
\hline 12 & -1.102 & -1.653 to -0.534 \\
\hline 13 & -0.345 & -0.792 to 0.111 \\
\hline 14 & 0.244 & -0.204 to 0.686 \\
\hline 15 & 0.160 & -0.283 to 0.599 \\
\hline 16 & -0.345 & -0.792 to 0.111 \\
\hline 17 & 0.581 & 0.099 to 1.049 \\
\hline 18 & 1.001 & 0.452 to 1.533 \\
\hline 19 & -0.682 & -1.163 to -0.186 \\
\hline 20 & -0.682 & -1.163 to -0.186 \\
\hline$N=20$ & & \\
\hline
\end{tabular}


Figures

Figure 1. The multi-component working memory model. Unshaded areas are representative of capacities which are 'fluid', such as temporary storage. The shaded areas represent cognitive systems which are 'crystallised' and accumulate long-term knowledge. Adapted from "The episodic buffer: A new component of working memory?" by A. Baddeley, 2000, Trends in Cognitive Sciences, 4, p. 421.

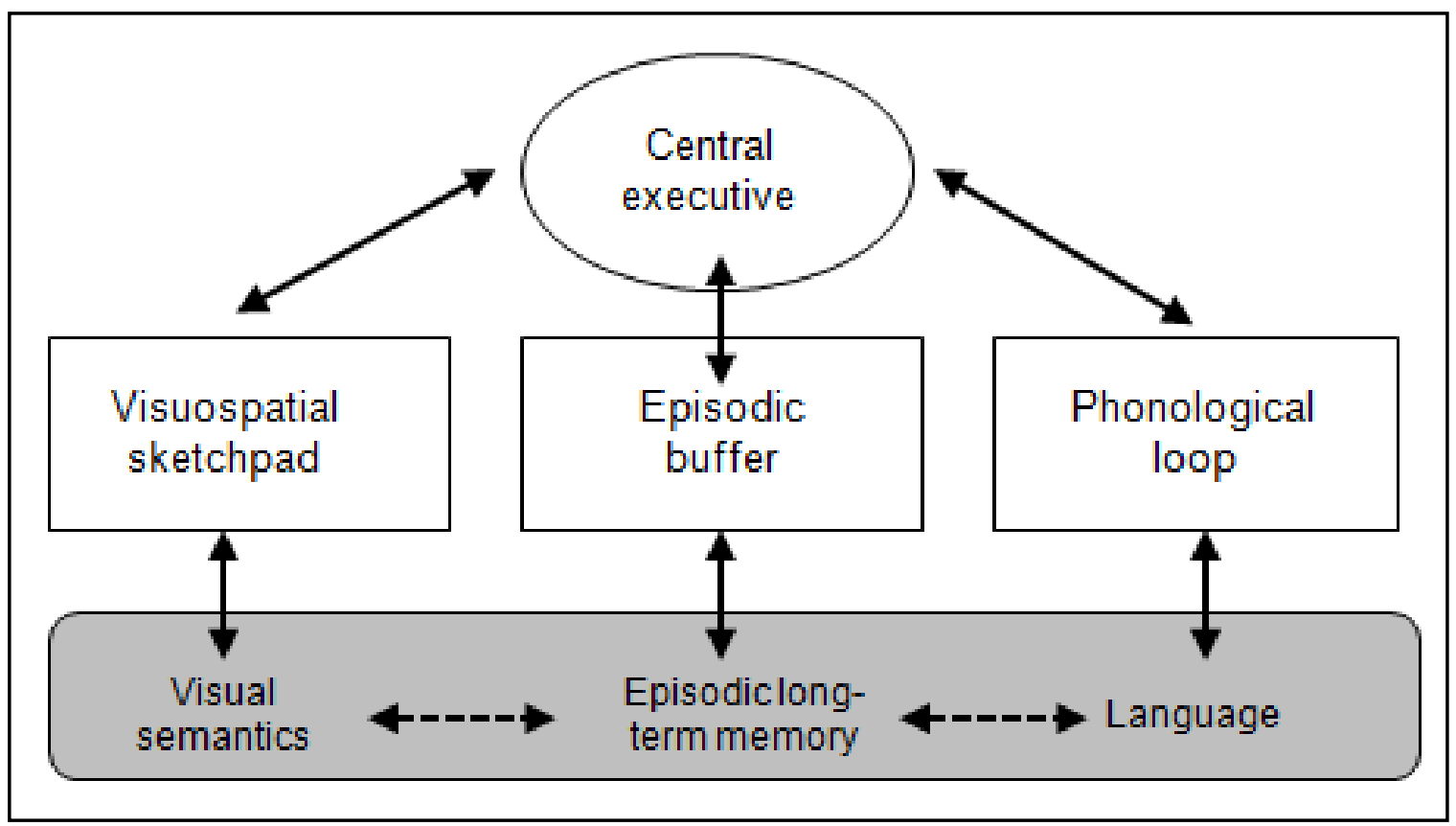

